\title{
A Modified Resource Distribution Fairness Measure
}

\author{
Zhenmin Chen \\ Department of Mathematics and Statistics, Florida International University, Miami, FL 33199, USA \\ Correspondence should be addressed to Zhenmin Chen; chenzh@fiu.edu
}

Received 19 August 2012; Revised 3 October 2012; Accepted 1 November 2012

Academic Editor: Keqin Gu

Copyright (C) 2013 Zhenmin Chen. This is an open access article distributed under the Creative Commons Attribution License, which permits unrestricted use, distribution, and reproduction in any medium, provided the original work is properly cited.

An important issue of resource distribution is the fairness of the distribution. For example, computer network management wishes to distribute network resource fairly to its users. To describe the fairness of the resource distribution, a quantitative fairness score function $F\left(x_{1}, x_{2}, \ldots, x_{n}\right)$ was proposed in 1984 by Jain et al. The purpose of this paper is to propose a modified network sharing fairness function so that the users can be treated differently according to their priority levels. The mathematical properties are discussed. The proposed fairness score function $F^{*}\left(x_{1}, x_{2}, \ldots, x_{n} ; w_{1}, w_{2}, \ldots, w_{n}\right)$ keeps all the nice properties of $F\left(x_{1}, x_{2}, \ldots, x_{n}\right)$ and provides better performance when the network users have different priority levels.

\section{Introduction}

When a fixed number of users or receivers share limited amount of resource, the fairness of the distribution is always an important issue. The resource distribution can be of any kind such as social benefit resource distribution, manpower distribution, and computer network resource distribution. Suppose $n$ users share a certain amount of resource. Let $x_{1}, x_{2}, \ldots, x_{n}$ be the amounts of resource the users receive, respectively. Suppose all $n$ users have the same right to share the entire resource. Then the difference among the values $x_{1}, x_{2}, \ldots, x_{n}$ should not be too large. If the difference among $n$ values is too large, then it can be claimed that the distribution of the entire resource is unfair. Here certain rules have to be made to determine if the resource distribution is fair or unfair. To solve such a problem, there are two important steps. The first step is to find an appropriate quantitative measure, which is a function of $x_{1}, x_{2}, \ldots, x_{n}$, such that the quantitative measure can be used to describe the fairness of the resource distribution. The quantitative measure should increase when the resource distribution becomes fairer. On the other hand, it should decrease when the resource distribution becomes more and more unfair. The second step is to determine when one can conclude that the resource distribution is significantly unfair. The concept of statistical test can be adopted for this purpose. For a certain level of significance, one may conclude that the resource distribution is significantly unfair when the quantitative measure falls below some value. Such a value is called a critical value in statistical analysis. This paper focuses on modifying a commonly used fairness measure so that the modified fairness measure can better fit the real world applications.

In the past several decades, many research papers have been published in this area in the literature. Jain et al. [1] proposed a quantitative measure to assess the fairness of the network resource distribution. The fairness score function proposed by Jain et al. is defined as

$$
F\left(x_{1}, x_{2}, \ldots, x_{n}\right)=\frac{\left(\sum_{i=1}^{n} x_{i}\right)^{2}}{n\left(\sum_{i=1}^{n} x_{i}^{2}\right)} .
$$

As mentioned above, a fairness score function must possess some nice properties so that it can be used to describe the fairness of the resource distribution and can be used to determine whether or not the resource distribution is fair. In fact, the fairness score function $F\left(x_{1}, x_{2}, \ldots, x_{n}\right)$ defined in (1) does possess some nice properties. It can be shown easily that $1 / n \leq F\left(x_{1}, x_{2}, \ldots, x_{n}\right) \leq 1$ for any nonnegative $x_{1}, x_{2}, \ldots, x_{n}$. In the case that the distribution is completely unfair, that is, only one user occupies the entire resource while the other users do not receive any, the value of $F\left(x_{1}, x_{2}, \ldots, x_{n}\right)$ is $1 / n$. On the other hand, if the distribution is perfectly fair, that is, all the users share the entire resource equally, then the value of $F\left(x_{1}, x_{2}, \ldots, x_{n}\right)$ is 1 . If only $k$ out of $n$ users share the entire resource equally 
while the others do not receive any, then the fairness score is $k / n$. It can also be seen that $F\left(x_{1}, x_{2}, \ldots, x_{n}\right)$ does not depend on scale. In addition, one can find that this fairness score function continuously reflects changes in allocation. For more references in this field, see Bertsekas and Gallager [2], Chiu and Jain [3], Kelly et al. [4], and Mazumdar et al. [5].

Note that the fairness score function of resource distribution can be used when all the users have equal right to share the entire resource. In practice, however, it is possible that the users may have different priority levels. For example, when the internet resource is distributed to users, advanced users and regular users should be treated differently. When social benefit resource is distributed, disabled people, pregnant women, low income, or no income people all have different priority levels. Equally distributing the entire resource to all the users is, in fact, an unfair way to distribute the entire resource. Therefore, some modification is needed to the fairness score function defined in (1) so that the modified fairness score function can be used for the case that users have different priority levels to share the entire resource. This is the purpose of this paper. Instead of equally distributing network resource to all the users, the entire resource is distributed to all users according to their priority levels. It can be shown that the fairness function proposed in this paper keeps all the meritorious properties of $F\left(x_{1}, x_{2}, \ldots, x_{n}\right)$ even for the case that the users are at different priority levels.

\section{Modified Fairness Score Function}

Now suppose the users who are sharing the entire resource have different priority levels. More specifically, let $x_{1}, x_{2}, \ldots, x_{n}$ be the amounts of resource that the users receive, respectively. Also let $w_{1}, w_{2}, \ldots, w_{n}\left(w_{i}>0, i=\right.$ $1,2, \ldots, n)$ be the corresponding priority factors of these users. It means that the amounts of resource users receive are supposed to be $w_{1} x, w_{2} x, \ldots, w_{n} x$ for some $x>0$. The fairness score function proposed in this paper is defined as

$$
\begin{aligned}
& F^{*}\left(x_{1}, x_{2}, \ldots, x_{n} ; w_{1}, w_{2}, \ldots, w_{n}\right) \\
& \quad=\left(n \sum_{i=1}^{n}\left(\frac{x_{i}}{\sum_{j=1}^{n} x_{j}}-\frac{w_{i}}{\sum_{j=1}^{n} w_{j}}\right)^{2}+1\right)^{-1} .
\end{aligned}
$$

This fairness score function is a generalization of the fairness score function defined in (1). In the case that all the users are at the same priority level, that is,

$$
w_{1}=w_{2}=\cdots=w_{n} .
$$

the fairness score function $F^{*}\left(x_{1}, x_{2}, \ldots, x_{n} ; w_{1}, w_{2}, \ldots, w_{n}\right)$ becomes $F\left(x_{1}, x_{2}, \ldots, x_{n}\right)$ which is same as the one defined in (1). To use this fairness score function to describe the fairness of the resource distribution and to use statistical analysis to determine whether or not the resource distribution is fair, $F^{*}\left(x_{1}, x_{2}, \ldots, x_{n} ; w_{1}, w_{2}, \ldots, w_{n}\right)$ must possess some nice properties mentioned in the previous section. In fact, it will be shown that the fairness score function $F^{*}\left(x_{1}, x_{2}, \ldots, x_{n} ; w_{1}, w_{2}, \ldots, w_{n}\right)$ does possess the same meritorious properties as $F\left(x_{1}, x_{2}, \ldots, x_{n}\right)$ does. It can be seen from the definition of $F^{*}\left(x_{1}, x_{2}, \ldots, x_{n} ; w_{1}, w_{2}, \ldots, w_{n}\right)$ that

$$
0 \leq F^{*}\left(x_{1}, x_{2}, \ldots, x_{n} ; w_{1}, w_{2}, \ldots, w_{n}\right) \leq 1
$$

is always true. The two extreme cases, that are the perfectly fair distribution case and the completely unfair case, are presented in Theorems 1 and 2. In the perfectly fair distribution case, the fairness score function defined in (2) reaches its highest value. In the completely unfair distribution case, the fairness score function reaches its lowest value.

Theorem 1. $F^{*}\left(x_{1}, x_{2}, \ldots, x_{n} ; w_{1}, w_{2}, \ldots, w_{n}\right)=1$ if and only if the distribution is perfectly fair. Here perfectly fair distribution refers to the case that all the users share the entire network resource proportionally according to their priority levels.

Proof. By the definition of $F^{*}\left(x_{1}, x_{2}, \ldots, x_{n} ; w_{1}, w_{2}, \ldots, w_{n}\right)$,

$$
F^{*}\left(x_{1}, x_{2}, \ldots, x_{n} ; w_{1}, w_{2}, \ldots, w_{n}\right)=1
$$

if and only if

$$
\sum_{i=1}^{n}\left(\frac{x_{i}}{\sum_{j=1}^{n} x_{j}}-\frac{w_{i}}{\sum_{j=1}^{n} w_{j}}\right)^{2}=0
$$

This statement is true if and only if

$$
\begin{gathered}
\frac{x_{1}}{\sum_{j=1}^{n} x_{j}}-\frac{w_{1}}{\sum_{j=1}^{n} w_{j}}=\frac{x_{2}}{\sum_{j=1}^{n} x_{j}}-\frac{w_{2}}{\sum_{j=1}^{n} w_{j}} \\
=\cdots=\frac{x_{n}}{\sum_{j=1}^{n} x_{j}}-\frac{w_{n}}{\sum_{j=1}^{n} w_{j}}=0 .
\end{gathered}
$$

It is equivalent to

$$
\frac{x_{1}}{\sum_{j=1}^{n} x_{j}}=\frac{w_{1}}{\sum_{j=1}^{n} w_{j}}, \frac{x_{2}}{\sum_{j=1}^{n} x_{j}}=\frac{w_{2}}{\sum_{j=1}^{n} w_{j}}, \ldots, \frac{x_{n}}{\sum_{j=1}^{n} x_{j}}=\frac{w_{n}}{\sum_{j=1}^{n} w_{j}}
$$

This ends the proof of the theorem.

Theorem 2. $F^{*}\left(x_{1}, x_{2}, \ldots, x_{n} ; w_{1}, w_{2}, \ldots, w_{n}\right)$ is minimized if and only if the distribution is completely unfair. Here the case that the distribution is completely unfair is the one that only one user with the lowest priority level occupies the entire resource.

Proof. Sort $w_{1}, w_{2}, \ldots, w_{n}$ from the smallest to the largest. Let $w_{(i)}$ be the $i$ th smallest number $(i=1,2, \ldots, n)$. Without loss of generality, it can be assumed that

$$
w_{1}=\min \left\{w_{1}, w_{2}, \ldots, w_{n}\right\}
$$


Thus $w_{1}=w_{(1)}$. Then

$$
\begin{aligned}
\sum_{i=1}^{n}\left(\frac{x_{i}}{\sum_{j=1}^{n} x_{j}}-\frac{w_{i}}{\sum_{j=1}^{n} w_{j}}\right)^{2} \\
=\left(\frac{x_{1}}{\sum_{j=1}^{n} x_{j}}-\frac{w_{1}}{\sum_{j=1}^{n} w_{j}}\right)^{2}+\sum_{i=2}^{n}\left(\frac{x_{i}}{\sum_{j=1}^{n} x_{j}}-\frac{w_{i}}{\sum_{j=1}^{n} w_{j}}\right)^{2} \\
=\left(\frac{x_{1}}{\sum_{j=1}^{n} x_{j}}-\frac{w_{1}}{\sum_{j=1}^{n} w_{j}}\right)^{2} \\
+\sum_{i=2}^{n}\left[\left(\frac{x_{i}}{\sum_{j=1}^{n} x_{j}}-\frac{w_{1}}{\sum_{j=1}^{n} w_{j}}\right)+\left(\frac{w_{1}}{\sum_{j=1}^{n} w_{j}}-\frac{w_{i}}{\sum_{j=1}^{n} w_{j}}\right)\right]^{2} \\
=\left(\frac{x_{1}}{\sum_{j=1}^{n} x_{j}} \frac{w_{1}}{\sum_{j=1}^{n} w_{j}}\right)^{2}+\sum_{i=2}^{n}\left(\frac{x_{i}}{\sum_{j=1}^{n} x_{j}}-\frac{w_{1}}{\sum_{j=1}^{n} w_{j}}\right)^{2} \\
+\sum_{i=1}^{n}\left(\frac{x_{i}}{\sum_{j=1}^{n} x_{j}}-\frac{w_{1}}{\sum_{j=1}^{n} w_{j}}\right)^{2} \\
\left.+\sum_{i=2}^{n}\left(\frac{w_{1}}{\sum_{j=1}^{n} w_{j}}-\frac{w_{i}}{\sum_{j=1}^{n} w_{j}}\right)^{2}\left(\frac{x_{i}}{\sum_{j=1}^{n} x_{j}}-\frac{w_{1}}{\sum_{j=1}^{n} w_{j}}\right)\left(\frac{w_{1}+w_{i}}{\sum_{j=1}^{n} w_{j} w_{j}}-\frac{w_{1}}{\sum_{j=1}^{n} w_{j}}\right)^{n}-\frac{w_{i}}{\sum_{j=1}^{n} w_{j}}\right)_{j=1}^{w_{j} w_{j}} \\
+
\end{aligned}
$$

Define

$$
\begin{gathered}
P=\sum_{i=1}^{n}\left(\frac{x_{i}}{\sum_{j=1}^{n} x_{j}}-\frac{w_{1}}{\sum_{j=1}^{n} w_{j}}\right)^{2}, \\
\mathrm{Q}=\sum_{i=2}^{n}\left(\frac{w_{1}}{\sum_{j=1}^{n} w_{j}}-\frac{w_{i}}{\sum_{j=1}^{n} w_{j}}\right)\left(\frac{2 x_{i}}{\sum_{j=1}^{n} x_{j}}-\frac{w_{1}+w_{i}}{\sum_{j=1}^{n} w_{j}}\right) .
\end{gathered}
$$

Note that

$$
\begin{gathered}
P=\sum_{i=1}^{n}\left(\frac{x_{i}}{\sum_{j=1}^{n} x_{j}}\right)^{2}-\frac{2 w_{1}}{\sum_{j=1}^{n} w_{j}} \sum_{i=1}^{n}\left(\frac{x_{i}}{\sum_{j=1}^{n} x_{j}}\right)+\sum_{i=1}^{n}\left(\frac{w_{1}}{\sum_{j=1}^{n} w_{j}}\right)^{2}, \\
=\sum_{i=1}^{n}\left(\frac{x_{i}}{\sum_{j=1}^{n} x_{j}}\right)^{2}-\frac{2 w_{1}}{\sum_{j=1}^{n} w_{j}}+\sum_{i=1}^{n}\left(\frac{w_{1}}{\sum_{j=1}^{n} w_{j}}\right)^{2} .
\end{gathered}
$$

Since $w_{1}, w_{2}, \ldots, w_{n}$ are fixed numbers, then to maximize $P$, it is desired to maximize

$$
R=\sum_{i=1}^{n}\left(\frac{x_{i}}{\sum_{j=1}^{n} x_{j}}\right)^{2}
$$

Since

$$
\sum_{i=1}^{n}\left(\frac{x_{i}}{\sum_{j=1}^{n} x_{j}}\right)=1
$$

then $R$ is maximized if and only if one of the

$$
\frac{x_{i}}{\sum_{j=1}^{n} x_{j}} \quad(i=1, \ldots, n)
$$

values is 1 , and the rest are zeros.

On the other hand, note that

$$
\frac{w_{1}}{\sum_{j=1}^{n} w_{j}}-\frac{w_{i}}{\sum_{j=1}^{n} w_{j}} \leq 0 \quad(i=2,3, \ldots, n) .
$$

In order to maximize $\mathrm{Q}$,

$$
\frac{2 x_{i}}{\sum_{j=1}^{n} x_{j}}=0
$$

must be true for those term satisfying $w_{1}<w_{i}$ for $i=$ $2, \ldots, n$. It is equivalent to the condition that $x_{i}$ must be 0 for those terms satisfying $w_{1}<w_{i}$ for $i=2, \ldots, n$. It means that the amounts that are received by users, whose priority levels are not the lowest, must be zero. Combining the results of the above discussion, the proof follows.

For fixed priority levels $w_{1}, w_{2}, \ldots, w_{n}$, the lowest value the fairness score function defined in (2) can reach is

$$
\left[n+1+n \sum_{i=1}^{n}\left(\frac{w_{i}}{\sum_{j=1}^{n} w_{j}}\right)^{2}-\frac{2 n w_{(1)}}{\sum_{j=1}^{n} w_{j}}\right]^{-1} .
$$

In the case of $w_{1}=w_{2}=\cdots=w_{n}$, this quantity becomes $1 / n$. This result is the same as the one when the fairness score function defined in (1) is used, as expected. The next theorem deals with the case that $k$ users equally share the entire resource assuming that all the users are at the same priority level.

Theorem 3. If all the $n$ users are at the same priority level, and if only $k$ out of the $n$ users share the entire resource equally while the other $n-k$ users do not share any, then

$$
F^{*}\left(x_{1}, x_{2}, \ldots, x_{n} ; w_{1}, w_{2}, \ldots, w_{n}\right)=\frac{k}{n} .
$$


Proof. Without loss of generality, assume the first $k$ users receive the same amount, say, $x$, and the rest users do not receive any. Then

$$
\begin{aligned}
F^{*} & \left(x_{1}, x_{2}, \ldots, x_{n} ; w_{1}, w_{2}, \ldots, w_{n}\right) \\
& =\left(n \sum_{i=1}^{n}\left(\frac{x_{i}}{\sum_{j=1}^{n} x_{j}}-\frac{w_{i}}{\sum_{j=1}^{n} w_{j}}\right)^{2}+1\right)^{-1} \\
& =\left(n \left(\sum_{i=1}^{k}\left(\frac{x}{k x}-\frac{w}{\sum_{j=1}^{n} w}\right)^{2}\right.\right. \\
& \left.\left.+\sum_{i=k+1}^{n}\left(\frac{0}{n x}-\frac{w}{\sum_{j=1}^{n} w}\right)^{2}\right)+1\right)^{-1} \\
= & \left(n\left(\sum_{i=1}^{k}\left(\frac{1}{k}-\frac{1}{n}\right)^{2}+\sum_{i=k+1}^{n}\left(-\frac{1}{n}\right)^{2}\right)+1\right)^{-1}=\frac{k}{n} .
\end{aligned}
$$

The proof is done.

It should be noted that Theorem 3 only deals with a special case that all the users have the same priority level. One might wonder if $F^{*}\left(x_{1}, x_{2}, \ldots, x_{n} ; w_{1}, w_{2}, \ldots, w_{n}\right)=k / n$ can hold for the case that users have different priority levels and that only $k$ out of the $n$ users share the entire resource proportionally according to their priority levels while the other $n-k$ users do not share any. It fact, this is not the case. The following is a counter example. Suppose there are three users with priority levels 3, 2, and 1, respectively. Suppose also that the users priority levels 3 and 2 share the entire resource proportionally to their priority levels and the user with priority level 1 does not receive any share. Then

$$
\begin{aligned}
& F^{*}\left(x_{1}, x_{2}, x_{3} ; w_{1}, w_{2}, w_{3}\right) \\
& =\left(3 \times\left(\left(\frac{3}{5}-\frac{3}{6}\right)^{2}+\left(\frac{2}{5}-\frac{2}{6}\right)^{2}+\left(0-\frac{1}{6}\right)^{2}\right)+1\right)^{-1} \\
& =\frac{150}{169} \neq \frac{2}{3} .
\end{aligned}
$$

Theorem 4. For $\eta>0$, define

$$
\begin{aligned}
D^{*}(\eta)= & F^{*}\left(x_{1}, \ldots, x_{s}-\eta, \ldots, x_{t}\right. \\
& \left.+\eta, \ldots, x_{n} ; w_{1}, w_{2}, \ldots, w_{n}\right) \\
& -F^{*}\left(x_{1}, \ldots, x_{s}, \ldots, x_{t}, \ldots, x_{n} ; w_{1}, w_{2}, \ldots, w_{n}\right) .
\end{aligned}
$$

Then

$$
\begin{aligned}
& \left\{\begin{array}{l}
>0 \\
\text { if } \eta<\left[\left(\left(\frac{x_{s}}{\sum_{j=1}^{n} x_{j}}\right)-\left(\frac{w_{s}}{\sum_{j=1}^{n} w_{j}}\right)\right)\right.
\end{array}\right. \\
& \left.-\left(\left(\frac{x_{t}}{\sum_{j=1}^{n} x_{j}}\right)-\left(\frac{w_{t}}{\sum_{j=1}^{n} w_{j}}\right)\right)\right] \\
& \cdot\left(\sum_{j=1}^{n} x_{j}\right)^{-1} \\
& =0 \\
& \text { if } \eta=\left[\left(\left(\frac{x_{s}}{\sum_{j=1}^{n} x_{j}}\right)-\left(\frac{w_{s}}{\sum_{j=1}^{n} w_{j}}\right)\right)\right. \\
& D^{*}(\eta)\left\{\quad-\left(\left(\frac{x_{t}}{\sum_{j=1}^{n} x_{j}}\right)-\left(\frac{w_{t}}{\sum_{j=1}^{n} w_{j}}\right)\right)\right] \\
& \cdot\left(\sum_{j=1}^{n} x_{j}\right)^{-1} \\
& <0 \\
& \text { if } \eta>\left[\left(\left(\frac{x_{s}}{\sum_{j=1}^{n} x_{j}}\right)-\left(\frac{w_{s}}{\sum_{j=1}^{n} w_{j}}\right)\right)\right. \\
& \left.-\left(\left(\frac{x_{t}}{\sum_{j=1}^{n} x_{j}}\right)-\left(\frac{w_{t}}{\sum_{j=1}^{n} w_{j}}\right)\right)\right] \\
& \left(\sum_{j=1}^{n} x_{j}\right)^{-1}
\end{aligned}
$$

Proof. By the definition of $D^{*}(\eta)$, it suffices to show that

$$
\begin{gathered}
\left(\frac{x_{s}}{\sum_{j=1}^{n} x_{j}}-\frac{w_{s}}{\sum_{j=1}^{n} w_{j}}\right)^{2}+\left(\frac{x_{t}}{\sum_{j=1}^{n} x_{j}}-\frac{w_{t}}{\sum_{j=1}^{n} w_{j}}\right)^{2} \\
-\left(\frac{x_{s}-\eta}{\sum_{j=1}^{n} x_{j}}-\frac{w_{s}}{\sum_{j=1}^{n} w_{j}}\right)^{2}-\left(\frac{x_{t}-\eta}{\sum_{j=1}^{n} x_{j}}-\frac{w_{t}}{\sum_{j=1}^{n} w_{j}}\right)^{2}, \\
\frac{x_{s}-x_{t}-\eta}{\sum_{j=1}^{n} x_{j}}+\frac{w_{t}-w_{s}}{\sum_{j=1}^{n} w_{j}}
\end{gathered}
$$


have the same sign. This is true because

$$
\begin{aligned}
& \left(\frac{x_{s}}{\sum_{j=1}^{n} x_{j}}-\frac{w_{s}}{\sum_{j=1}^{n} w_{j}}\right)^{2}+\left(\frac{x_{t}}{\sum_{j=1}^{n} x_{j}}-\frac{w_{t}}{\sum_{j=1}^{n} w_{j}}\right)^{2} \\
& \quad-\left(\frac{x_{s}-\eta}{\sum_{j=1}^{n} x_{j}}-\frac{w_{s}}{\sum_{j=1}^{n} w_{j}}\right)^{2}-\left(\frac{x_{t}-\eta}{\sum_{j=1}^{n} x_{j}}-\frac{w_{t}}{\sum_{j=1}^{n} w_{j}}\right)^{2} \\
& =\left(\frac{2 x_{s}-\eta}{\sum_{j=1}^{n} x_{j}}-\frac{2 w_{s}}{\sum_{j=1}^{n} w_{j}}\right)\left(\frac{\eta}{\sum_{j=1}^{n} x_{j}}\right) \\
& +\left(\frac{2 x_{t}+\eta}{\sum_{j=1}^{n} x_{j}}-\frac{2 w_{t}}{\sum_{j=1}^{n} w_{j}}\right)\left(\frac{\eta}{\sum_{j=1}^{n} x_{j}}\right) \\
& =\eta \cdot\left[\left(\frac{2 x_{s}-\eta}{\sum_{j=1}^{n} x_{j}}-\frac{2 w_{s}}{\sum_{j=1}^{n} w_{j}}\right)-\left(\frac{2 x_{t}+\eta}{\sum_{j=1}^{n} x_{j}}-\frac{2 w_{t}}{\sum_{j=1}^{n} w_{j}}\right)\right] \\
& =(2 \eta) \cdot\left(\frac{x_{s}-x_{t}-\eta}{\sum_{j=1}^{n} x_{j}}+\frac{w_{t}-w_{s}}{\sum_{j=1}^{n} w_{j}}\right) .
\end{aligned}
$$

The proof of the theorem follows.

Theorem 4 shows that when user $i$ gives a small amount $\eta$ to user $j$, then the new allocation is more fair if user $j$ was relatively discriminated. The new allocation is less fair if user $j$ was relatively favored. The idea was adopted from Jain et al. [1] in conjunction with the consideration of the priority levels.

The following result shows that if all the users are given extra amounts of network resource proportionally according to their priority levels, then the fairness of the distribution will not decrease.

Theorem 5. For any $\delta>0$,

$$
\begin{gathered}
F^{*}\left(x_{1}+\frac{\delta w_{1}}{\sum_{j=1}^{n} w_{j}}, x_{2}+\frac{\delta w_{2}}{\sum_{j=1}^{n} w_{j}}, \ldots, x_{n}\right. \\
\left.\quad+\frac{\delta w_{n}}{\sum_{j=1}^{n} w_{j}} ; w_{1}, w_{2}, \ldots, w_{n}\right) \\
\geq F^{*}\left(x_{1}, x_{2}, \ldots, x_{n} ; w_{1}, w_{2}, \ldots, w_{n}\right) .
\end{gathered}
$$

Proof. It can be shown that

$$
\begin{aligned}
F^{*}\left(x_{1}+\frac{\delta w_{1}}{\sum_{j=1}^{n} w_{j}}, x_{2}+\frac{\delta w_{2}}{\sum_{j=1}^{n} w_{j}}, \ldots, x_{n}\right. \\
\left.\quad+\frac{\delta w_{n}}{\sum_{j=1}^{n} w_{j}} ; w_{1}, w_{2}, \ldots, w_{n}\right) \\
=\left[n \sum_{i=1}^{n}\left(\frac{x_{i}+\left(\delta w_{i} / \sum_{j=1}^{n} w_{j}\right)}{\sum_{j=1}^{n} x_{j}+\delta}-\frac{w_{i}}{\sum_{j=1}^{n} w_{j}}\right)^{2}\right]^{-1}
\end{aligned}
$$

Now it is desired to show that

$$
\sum_{i=1}^{n}\left(\frac{x_{i}+\left(\delta w_{i} / \sum_{j=1}^{n} w_{j}\right)}{\sum_{j=1}^{n} x_{j}+\delta}-\frac{w_{i}}{\sum_{j=1}^{n} w_{j}}\right)^{2},
$$

is a decreasing function in $\delta$. This is true because

$$
\begin{aligned}
& \sum_{i=1}^{n}\left(\frac{x_{i}+\left(\delta w_{i} / \sum_{j=1}^{n} w_{j}\right)}{\sum_{j=1}^{n} x_{j}+\delta}-\frac{w_{i}}{\sum_{j=1}^{n} w_{j}}\right)^{2} \\
& \quad=\sum_{i=1}^{n}\left[\frac{\left(x_{i}+\left(\delta w_{i} / \sum_{j=1}^{n} w_{j}\right)\right)-\left(w_{i} / \sum_{j=1}^{n} w_{j}\right)\left(\sum_{j=1}^{n} x_{j}+\delta\right)}{\sum_{j=1}^{n} x_{j}+\delta}\right]^{2} \\
& \quad=\sum_{i=1}^{n}\left[\frac{x_{i}-\left(w_{i} / \sum_{j=1}^{n} w_{j}\right)\left(\sum_{j=1}^{n} x_{j}\right)}{\sum_{j=1}^{n} x_{j}+\delta}\right]^{2}
\end{aligned}
$$

The proof follows.

In addition to the above properties, it can be seen easily the fairness function defined in (2) does not depend on scale. The properties of the fairness score function ensures that the fairness score function can be used to evaluate the fairness of the resource distribution.

\section{Conclusion and Discussion}

Fairness is an important issue when resource is distributed to its users. Here resource distribution can be of any kind. For example, when internet resource is distributed to users, one wishes to distribute the entire resource fairly to the users. Social benefit resource distribution is another example. The fairness measure of the resource distribution was originally discussed in the area of computer network resource distribution. The fairness score function, $F\left(x_{1}, x_{2}, \ldots, x_{n}\right)$, proposed by Jain et al. [1] has been widely used to evaluate the fairness of the network resource distribution. The fairness score function $F\left(x_{1}, x_{2}, \ldots, x_{n}\right)$, however, assumes that all the users are at the same priority level; that is, all the users are supposed to be treated equally when the entire resource is distributed. In practice, users may have different priority levels and should be treated differently. When computer network resource providers distribute the entire resource to their users, they usually treat their users according to their priority levels-basic users, advanced users, VIP users, and so on. Social benefit resource is also distributed to the receivers according to their priority levels. For example, disabled people usually have higher priority to receive social benefits. Therefore, some modification is needed on the fairness score function so that the modified fairness score function can handle the case that the users have different priority levels. This is the purpose of this paper. A new fairness score function $F^{*}\left(x_{1}, x_{2}, \ldots, x_{n} ; w_{1}, w_{2}, \ldots, w_{n}\right)$ is presented in this paper. The fairness score function proposed in this paper keeps all the nice properties of $F\left(x_{1}, x_{2}, \ldots, x_{n}\right)$ even when the users have different priority levels.

The value of the new fairness score function $F^{*}\left(x_{1}\right.$, $\left.x_{2}, \ldots, x_{n} ; w_{1}, w_{2}, \ldots, w_{n}\right)$ increases when the resource distribution becomes fairer and fairer. The value decreases when the resource distribution becomes more and more unfair. One extreme of the resource distribution is that all the users share the entire resource according to their 
priority levels. In that case, the fairness score function $F^{*}\left(x_{1}, x_{2}, \ldots, x_{n} ; w_{1}, w_{2}, \ldots, w_{n}\right)$ reaches its maximum value 1 . On the other hand, when the resource distribution is the most unfair case, the fairness score function $F^{*}\left(x_{1}, x_{2}, \ldots, x_{n} ; w_{1}, w_{2}, \ldots, w_{n}\right)$ reaches its minimum value. Furthermore, the status of fairness distribution changes from one extreme to the other extreme, the fairness score function $F^{*}\left(x_{1}, x_{2}, \ldots, x_{n} ; w_{1}, w_{2}, \ldots, w_{n}\right)$ is able to reflect the change correspondingly. These properties ensure that the fairness score function $F^{*}\left(x_{1}, x_{2}, \ldots, x_{n} ; w_{1}, w_{2}, \ldots, w_{n}\right)$ can be used to evaluate the fairness of the resource distribution.

It can be shown that the distribution of $F^{*}\left(x_{1}, x_{2}\right.$, $\left.\ldots, x_{n} ; w_{1}, w_{2}, \ldots, w_{n}\right)$ is scale-free. Thus the quantiles of $F^{*}\left(x_{1}, x_{2}, \ldots, x_{n} ; w_{1}, w_{2}, \ldots, w_{n}\right)$ can be obtained by MonteCarlo simulation. Based on those quantiles, a statistical test can then be conducted to check whether or not the network resource distribution is statistically significantly unfair to the users. In computer network resource management, if the resource distribution is significantly unfair, the resource distribution scheme should be adjusted.

\section{Acknowledgment}

The author thanks two anonymous referees for their detailed comments and suggestions that greatly improved the quality of the paper.

\section{References}

[1] R. Jain, D. Chiu, and W. Hawe, "A quantitative measure of fairness and discrimination for resource allocation in shared computer system," Technical Report DEC-TR-301, Digital Equipment Corporation, Maynard, Mass, USA, 1984.

[2] D. Bertsekas and R. Gallager, Data Networks, Prentice Hall, Upper Saddle River, NJ, USA, 1987.

[3] D. M. Chiu and R. Jain, "Analysis of the increase and decrease algorithms for congestion avoidance in computer networks," Computer Networks and ISDN Systems, vol. 17, no. 1, pp. 1-14, 1989.

[4] F. P. Kelly, A. K. Maulloo, and D. Tan, "Rate control for communication networks: shadow prices, proportional fairness and stability," Journal of the Operational Research Society, vol. 49, no. 3, pp. 237-252, 1998.

[5] R. Mazumdar, L. G. Mason, and C. Douligeris, "Fairness in network optimal flow control: optimality of product forms," IEEE Transactions on Communications, vol. 39, no. 5, pp. 775-782, 1991. 


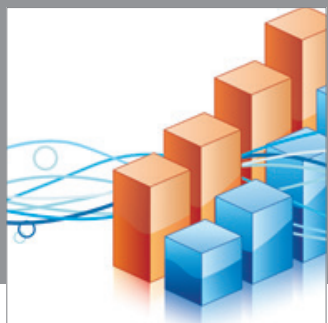

Advances in

Operations Research

mansans

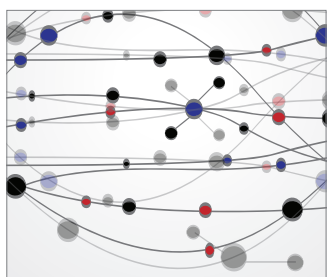

The Scientific World Journal
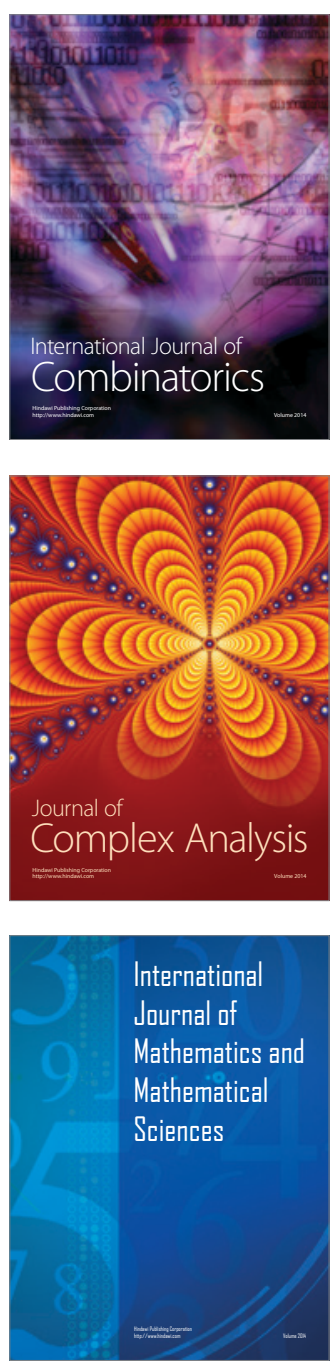
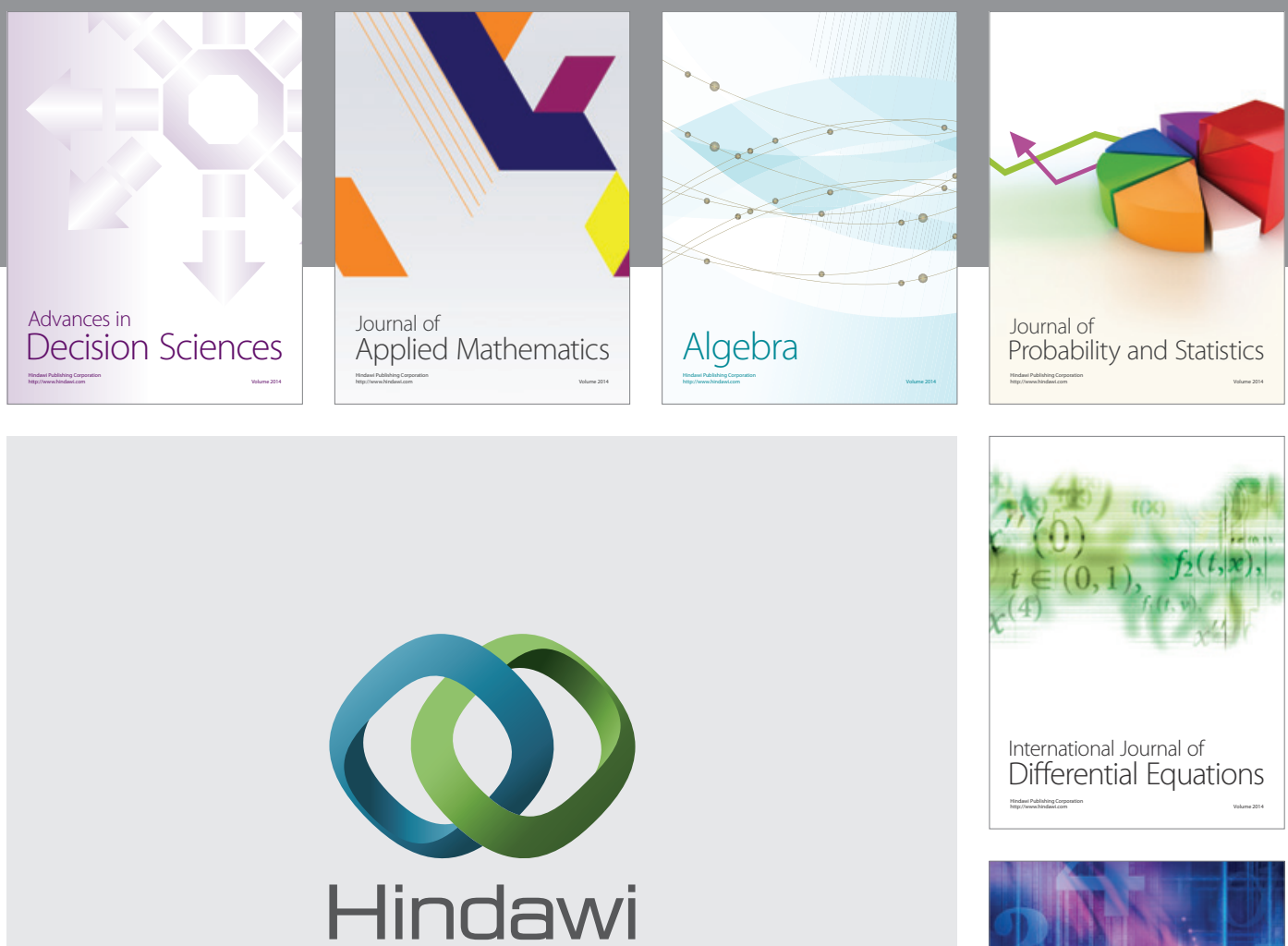

Submit your manuscripts at http://www.hindawi.com
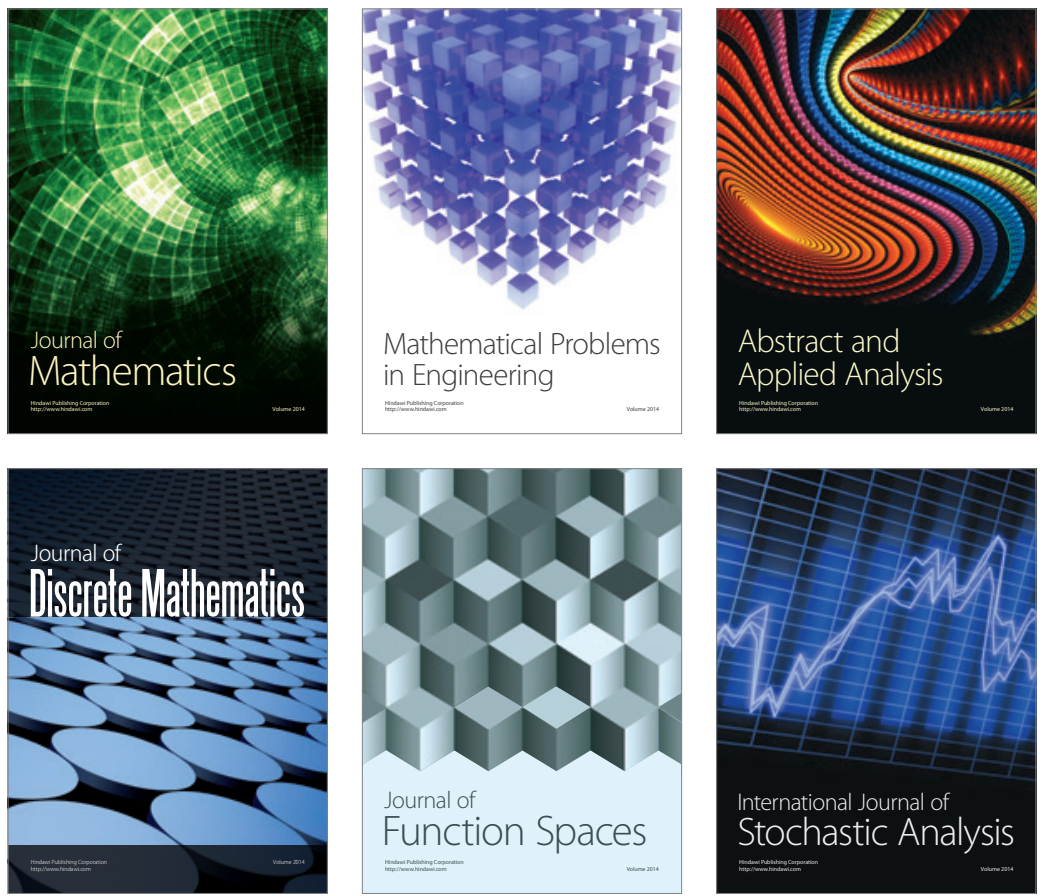

Journal of

Function Spaces

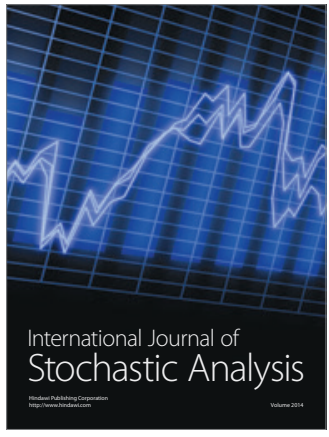

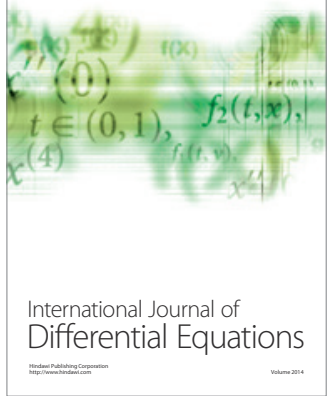
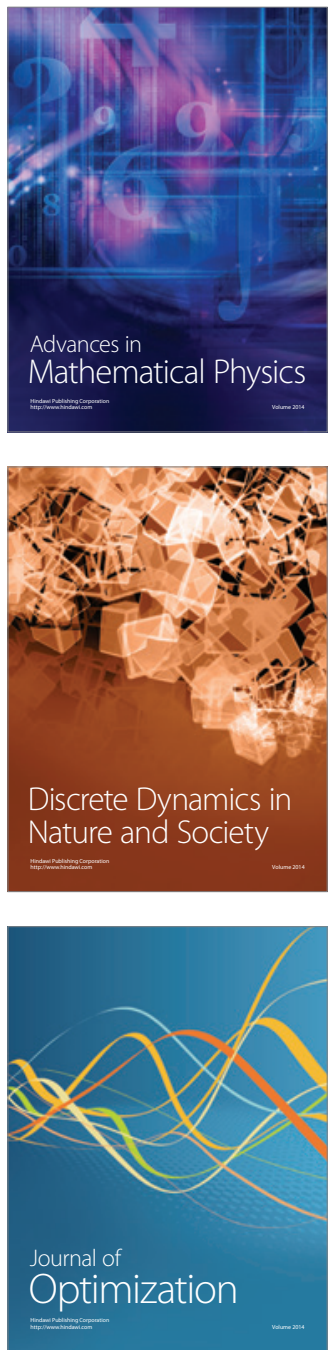